\title{
Segmentation by Adaptive Geodesic Active Contours
}

\author{
Carl-Fredrik Westin ${ }^{1}$, Liana M. Lorigo ${ }^{2}$, Olivier Faugeras ${ }^{2,3}$, \\ W. Eric L. Grimson ${ }^{2}$, Steven Dawson ${ }^{4}$, Alexander Norbash ${ }^{1}$, and Ron Kikinis ${ }^{1}$ \\ 1 Harvard Medical School, Brigham \& Women's Hospital, Boston MA, USA \\ 2 MIT Artificial Intelligence Laboratory, Cambridge, MA USA \\ 3 INRIA, Sophia Antipolis, France \\ 4 Harvard Medical School, Mass. General Hospital, Boston MA, USA \\ westin@bwh.harvard.edu
}

\begin{abstract}
This paper introduces the use of spatially adaptive components into the geodesic active contour segmentation method for application to volumetric medical images. These components are derived from local structure descriptors and are used both in regularization of the segmentation and in stabilization of the image-based vector field which attracts the contours to anatomical structures in the images. They are further used to incorporate prior knowledge about spatial location of the structures of interest. These components can potentially decrease the sensitivity to parameter settings inside the contour evolution system while increasing robustness to image noise. We show segmentation results on blood vessels in magnetic resonance angiography data and bone in computed tomography data.
\end{abstract}

\section{Introduction}

Curve-shortening flow is the evolution of a curve over time to minimize some distance metric. When this distance metric is based on image properties, it can be used for segmentation. The idea of geodesic active contours is to define the metric so that indicators of the object boundary, such as large intensity gradients, have a very small "distance" $[4,9]$. The minimization will attract the curve to such image areas, thereby segmenting the image, while preserving properties of the curve such as smoothness and connectivity. Geodesic active contours can also be viewed as a more mathematically sophisticated variant of classical snakes [8]. Further, they are implemented with level set methods [15] which are based on recent results in differential geometry $[6,5]$. The method can be extended to evolve surfaces in $3 \mathrm{D}$, for the segmentation of $3 \mathrm{D}$ imagery such as medical datasets [4, 22]. More recent work developed the level set equations necessary to evolve arbitrary dimensional manifolds in arbitrary dimensional space $[1,13]$.

This paper introduces the use of spatially adaptive components into the geodesic active contour segmentation framework. These components are derived from local structure descriptors and are used both in regularization of the segmentation and in stabilization of the image-based vector field which attracts 
the contours to anatomical structures in the images. Local structure can be described in terms of how similar an image region is to a plane, to a line, and to a sphere. Describing the signal in geometrical terms rather than as a neighborhood of voxel values is intuitively appealing. We have applied a tensor-based description method for estimating these geometrical properties to the problem of segmenting bone in computed tomography (CT) data, with a focus on thin bone, which is especially difficult to obtain [18].

\section{Background}

Local image structure properties can be estimated from derivatives of the intensity image or the responses of directed quadrature filters. We will incorporate them into the geodesic active contour framework, a powerful segmentation technique.

\subsection{Local Image Structure}

In two dimensions, local structure estimation has been used to detect and describe edges and corners [7]. The local structure is described in terms of dominant local orientation and isotropy, where isotropy means lack of dominant orientation. In three dimensions, local structure has been used to describe landmarks, rotational symmetries, and motion $[10,2,11,14,16]$. In addition to isotropy, it describes geometrical properties which have been applied to the enhancement and segmentation of blood vessels in volumetric angiography datasets $[12,17$, bone in CT images [21, 20], and to the analysis of white matter in diffusion tensor magnetic resonance imaging [19].

Let the operator $\sum_{\Omega}$ denote averaging in the local neighborhood $\Omega$ about the current spatial location, and assume $3 \mathrm{D}$ data. Then the eigenvalues $\lambda_{1} \geq \lambda_{2} \geq \lambda_{3}$ of the tensor

$$
T_{\Omega}=\frac{\sum_{\Omega} \nabla I \nabla I^{T}}{\sum_{\Omega}|\nabla I|^{2}}
$$

describe geometrical properties over the neighborhood $\Omega$, such as the following generic cases:

1. $\lambda_{1}=1, \lambda_{2}=\lambda_{3}=0$, when the signal is locally planar.

2. $\lambda_{1}=\lambda_{2}=1, \lambda_{3}=0$, when the signal is locally tubular.

3. $\lambda_{1}=\lambda_{2}=\lambda_{3}=1$, in regions that contain intensity gradients but no dominant orientational structure.

From these cases, we can define scalar measures indicating which local structure is present. An example measure of similarity to thin tubular shapes is

$$
c_{\text {linear }}=\frac{\lambda_{2}-\lambda_{3}}{\lambda_{1}} .
$$

In higher dimensions, the basic shapes are more complicated than lines and planes, and the possible anisotropies become more complex. 


\subsection{Geodesic Active Contours, Level Set Methods}

The task of finding the curve that best fits an object boundary is posed as a minimization problem over all closed planar curves $C(p):[0,1] \rightarrow \mathbb{R}^{2}[4,3,9]$. The objective function is

$$
\oint_{0}^{1} g(|\nabla I(C(p))|)\left|C^{\prime}(p)\right| d p
$$

where $I$ is the image and $g$ is a strictly decreasing function that approaches zero as image gradients become large. To minimize this weighted curve length by steepest descent, one uses the evolution equation

$$
C_{t}=(g \kappa-\nabla g \cdot \hat{n}) \hat{n}
$$

where $C_{t}$ is the derivative of $C$ with respect to an artificial time parameter $t, \kappa$ is the Euclidean curvature, and $\hat{n}$ is the unit inward normal.

Level set methods increase the dimensionality of the problem from the dimensionality of the evolving manifold to the dimensionality of the embedding space to achieve independence of parameterization and topological flexibility $[6,5,15]$. For the example of planar curves, instead of evolving the one-dimensional curve, the method evolves a two-dimensional surface. Let $u$ be the signed distance function to curve $C$; that is, the value of $u$ at each point is the distance to the closest point on $C$, with interior points designated by negative distances. Consequently, $C$ is the zero level-set of $u$. Then evolving $C$ according to Equation 3 is equivalent to evolving $u$ according to

$$
u_{t}=g \kappa|\nabla u|+\nabla g \cdot \nabla u
$$

in the sense that the zero level set of $u$ remains the evolving curve $C$ over time. The extension to surfaces in 3D is straightforward and is called minimal surfaces $[4]$.

Now presume $u$ is the signed distance function to a surface $S$ in 3D. Expanding $\nabla g$ using the chain rule and replacing $\kappa|\nabla u|$ with a more general regularization term $\lambda$ gives

$$
u_{t}=g \lambda+g^{\prime} \nabla u \cdot H \frac{\nabla I}{|\nabla I|} .
$$

The codimension of a manifold is the difference between the dimension of the embedding space and the dimension of the manifold. We will say that a regularization force for an evolving surface is "codimension-two" if it is based on the smaller principal curvature of the surface; for a tubular surface, this curvature approximates the Euclidean curvature of the centerline of the tube which has codimension two. We will say it is "codimension-one" if it is based on the mean curvature of the surface. If $p_{1} \geq p_{2}$ are the principal curvatures of the surface, then these forces are given by

\begin{tabular}{|c|c|}
\hline codimension-one force & codimension-two force \\
\hline$\lambda=\frac{p_{1}+p_{2}}{2}|\nabla u|$ & $\lambda=p_{2}|\nabla u|$ \\
\hline
\end{tabular}


Let $\lambda_{1} \geq \lambda_{2}$ be the eigenvalues of the operator

$$
F=P_{\nabla u} \nabla^{2} u P_{\nabla u}=\left(I_{d}-\frac{\nabla u \nabla u^{T}}{|\nabla u|^{2}}\right) H_{u}\left(I_{d}-\frac{\nabla u \nabla u^{T}}{|\nabla u|^{2}}\right)
$$

where $I_{d}$ is the identity matrix and $P_{\nabla u}$ is the projector onto the plane normal to $\nabla u$. It turns out that $\lambda_{1}=p_{1}|\nabla u|$ and $\lambda_{2}=p_{2}|\nabla u|$, in the limiting case.

In the next section we describe how the local structure tensor (Equation 1) can be incorporated into the geodesic active contour equation (Equation 4). The basic idea that is explored in this paper is how to perform codimension-one regularization in image regions that are similar to a plane, and codimension-two regularization in regions similar to a line.

\section{Adaptive Geodesic Active Contours}

Observe that the evolution (Equation 4) is based on two terms: (1) a regularization term which ensures smoothness of the evolved surface, and (2) an image term that defines an vector field that is attracting the surface to the image structures of interest. We apply local structure estimation to adaptively modify each of these terms.

\subsection{Adaptive Regularization Force}

To evolve surfaces in 3D, the minimal surfaces work [4] uses the mean curvature of the surface for regularization, which corresponds to the "codimension-one force". When one wishes to evolve tubular surfaces, the smaller principal curvature is more appropriate to avoid eliminating the high curvatures inherent in the tube-like shape [13], which corresponds to the "codimension-two force."

To illustrate the difference, we created a test volume containing a thin tube connected to a flat sheet. We corrupted this image with a high level of noise. Figure 1 illustrates the result of regularizing this shape using the two different forces. The first image shows the initial shape, obtained by thresholding the volume. The second shows the result of applying the codimension-one force and the third the codimension-two force. Notice that the codimension-two force retained the thin tube while reducing the noise along it, while the codimension-one force deleted the tube but obtained a smoother plane than did the codimensiontwo force. In both experiments we iterated the evolution equations 20 times. It should be mentioned that with more iterations the result of the codimensiontwo does get smoother. However our comparison shows that the codimension-one regularization converges faster and therefor is assumed to be more resistant to noise.

This experiment illustrates the potential benefit of choosing the regularization force locally, dependent on the shape of the object to be segmented. One would expect to benefit by choosing a continuous value between the smaller curvature and the mean curvature. We propose to select this value according to the 


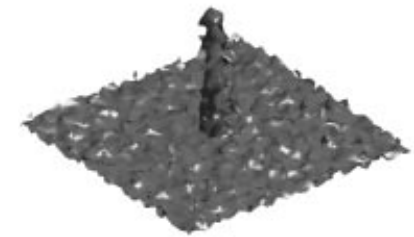

Initial surface

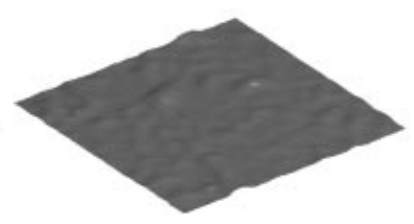

Codimension-one regularization

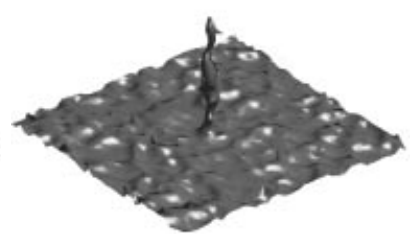

Codimension-two regularization

Fig. 1. Illustration of difference between codimension-one regularization force and codimension-two regularization force: initial surface, result of codimensionone, and result of codimension-two.

local structure estimates. The evolution equation (Equation 5) becomes

$$
u_{t}=g\left(\gamma \lambda_{2}+(1-\gamma) \frac{\lambda_{1}+\lambda_{2}}{2}\right)+g^{\prime} \nabla u \cdot H \frac{\nabla I}{|\nabla I|}
$$

where $\gamma$ is a scalar between 0 and 1 indicating how planar or how tubular the local image structure is. Setting $\gamma=c_{\text {linear }}$ (Equation 2) gives the desired result of $\gamma=1$ for codimension-two regularization of tubular objects and $\gamma=0$ for codimension-one regularization of planar objects and those without dominant orientational structure.

Referring back to Equation 6 and noting that the trace operation yields the sum of the eigenvalues, we obtain another formulation for the mean curvature

$$
\frac{\lambda_{1}+\lambda_{2}}{2}=\frac{1}{2|\nabla u|} \operatorname{trace}\left(\left(I_{d}-\frac{\nabla u \nabla u^{T}}{|\nabla u|^{2}}\right) H_{u}\left(I_{d}-\frac{\nabla u \nabla u^{T}}{|\nabla u|^{2}}\right)\right) .
$$

Close to the correct segmentation result, $\nabla u$ should be approximately parallel to the image gradient $\nabla I$, so one could project out the $\nabla I$ direction instead of the $\nabla u$ direction:

$$
\frac{\lambda_{1}+\lambda_{2}}{2} \approx \frac{1}{2|\nabla u|} \operatorname{trace}\left(\left(I_{d}-\frac{\nabla I \nabla I^{T}}{|\nabla I|^{2}}\right) H_{u}\left(I_{d}-\frac{\nabla I \nabla I^{T}}{|\nabla I|^{2}}\right)\right) .
$$

The tensor $\frac{\nabla I \nabla I^{T}}{|\nabla I|^{2}}$ is a projector onto the direction $\nabla I$; likewise, $I_{d}-\frac{\nabla I \nabla I^{T}}{|\nabla I|^{2}}$ is the projector that removes all components in the direction $\nabla I$. By local averaging of the image-based projectors according to Equation 1, we obtain

$$
\lambda_{2} \approx \frac{1}{|\nabla u|} \operatorname{trace}\left(\left(I_{d}-T_{\Omega}\right) H_{u}\left(I_{d}-T_{\Omega}\right)\right) .
$$

The local averaging acts to remove all components in all directions around the curve, leaving only those components in the tangent direction. Thus, we have an approximation to the curvature of the curve, which is the smaller principal curvature of the thin tube around it. Note that we have $\lambda=\lambda_{2}$ for codimension-two, 

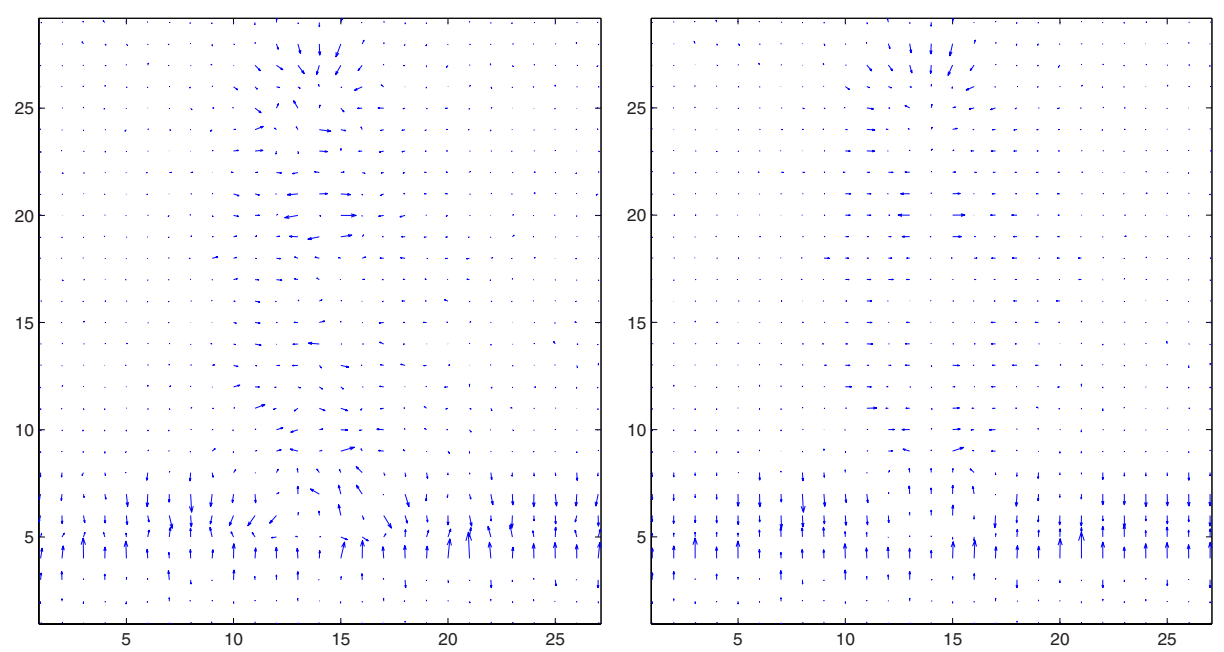

Fig. 2. Visualization of auxiliary vector field. For one slice through the center of our test volume, we show the raw vector field $H \frac{\nabla I}{|\nabla I|}$ (left) and the stabilized vector field $T_{\Omega} H \frac{\nabla I}{|\nabla I|}$ (right).

and for codimension-one $\lambda=\lambda_{1}+\lambda_{2}$. When image noise is high, however, the image gradient estimations will often be less stable than using curvature estimates of the regularized embedding function $u$. For this reason, our implementation uses Equation 7.

\subsection{Adaptive Image Force}

Local Image Structure For structures such as vessels in magnetic resonance angiography (MRA) and bronchi CT which appear brighter than the background, a weight on the image term defined by the cosine of the angle between the normal to the surface and the gradient in the image was introduced in [13]. This cosine is given by the dot product of the respective gradients of $u$ and $I$, so the update equation (Equation 5) becomes

$$
u_{t}=g \lambda+g^{\prime}\left(-\frac{\nabla u}{|\nabla u|} \cdot \frac{\nabla I}{|\nabla I|}\right) \nabla u \cdot H \frac{\nabla I}{|\nabla I|} .
$$

Similar to the equality $\left(a^{T} b\right)\left(a^{T} b\right)=a a^{T} \cdot b b^{T}=A \cdot B$ where $A$ and $B$ are matrices and the dot product of matrices is defined as the sum of the elementwise products, we can write

$$
u_{t}=g \lambda-g^{\prime} \frac{\nabla u \nabla u^{T}}{|\nabla u|} \cdot H \frac{\nabla I \nabla I^{T}}{|\nabla I|^{2}} .
$$




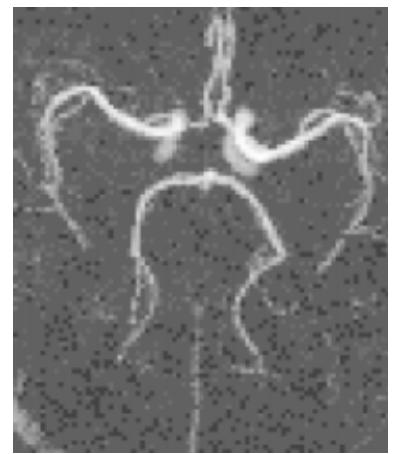

MIP

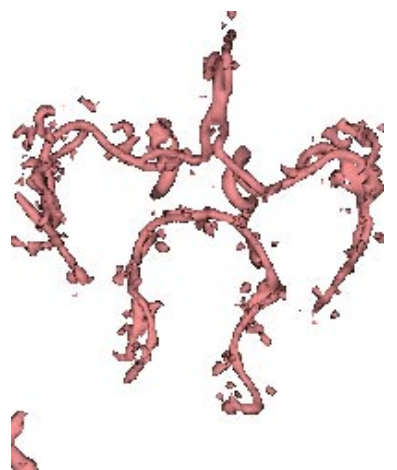

Codimension-two regularization

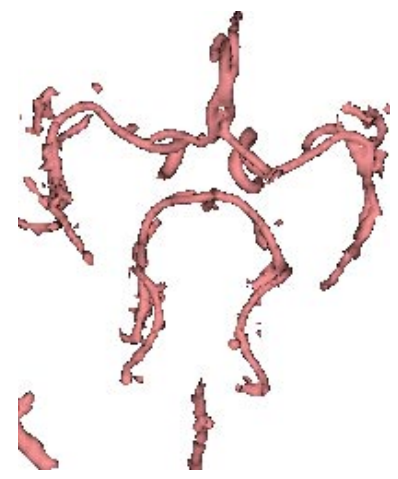

Adaptive codimension regularization

Fig. 3. The maximum intensity projection (MIP) of an MRA data set of the brain followed by the segmentation obtained with the original auxiliary image vector term and that obtained with adaptive codimension regularization and stabilized image vector term. Notice that the latter captures more vessels while demonstrating increased resistance to noise.

Recall that $g$ must be decreasing function that approaches zero on large image gradients. Choosing $g=\exp (-|\nabla I|)$ as in [13] implies that $\frac{g}{g^{\prime}}=-1$. Divide both components of the evolution by $g$ to obtain

$$
u_{t}=\lambda+\frac{\nabla u \nabla u^{T}}{|\nabla u|} \cdot H \underbrace{\frac{\nabla I \nabla I^{T}}{|\nabla I|^{2}}}_{T}
$$

We observe again the projection operator $T$ as in Equation 9, similar to the differential operator in Equation 1. The same arguments apply: $T$ alone projects onto the direction of the gradient $\nabla I$, but when smoothed, $T_{\Omega}$ has an adaptive behavior. When all gradients in the neighborhood are similar, as in the case of a plane, it projects as $T$, but when the gradients vary around a thin tubular structure, it projects to the plane orthogonal to the centerline of this structure. One could replace $T$ with $T_{\Omega}$ in Equation 13 to achieve this behavior.

The same intuition can be used, however, to modify the current evolution equation (Equation 11) instead. Since we are interested in only the component of the evolution that is normal to object surface, it is desirable to have stable gradients in this direction; the derived projection operator will have this stabilizing effect. The evolution equation now becomes

$$
u_{t}=\kappa|\nabla u|+\left(\frac{\nabla u \nabla u^{T}}{|\nabla u|} \cdot \frac{\nabla I}{|\nabla I|}\right) \cdot T_{\Omega} H \frac{\nabla I}{|\nabla I|} .
$$

The operator $T_{\Omega}$ can be viewed as an adaptive relaxation of the image-based (auxiliary) vector field based on local image constraints, as illustrated in Figure 


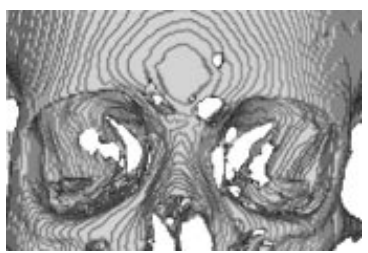

Initial surface

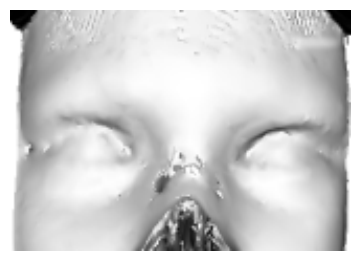

Codimension-one regularization

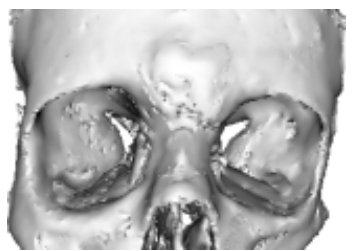

Spatially constrained adaptive codimension

Fig. 4. Incorporation of spatial priors into the auxiliary vector field, in conjunction with an active contour model. The surface used to initialize the evolution is shown first, followed by the results of the unconstrained evolution and of the constrained evolution.

2. Notice that the noisy gradients along both the plane and the stick become much closer to desired intensity gradients, perpendicular to the image structures. In areas of well-defined gradients the operator $T_{\Omega}$ acts like a projection operator. In unstructured areas the operator will be close to the identity operator.

Figure 3 compares the segmentations obtained by Equation 11 with those obtained by Equation 14 for a cerebral MRA dataset. We corrupted the image with synthetic noise to demonstrate the stabilization of the auxiliary vector field. Notice that the segmentation obtained using the adaptive codimension is smoother without loosing important details which is consistent with our intuition gained from Figure 1 where codimension-one regularization gives a smoother surface, and codimension-two regularization preserves tubular structures.

Incorporating External Models External spatial models can also be incorporated through the auxiliary vector field. We represent prior knowledge about the possible locations of the objects to be segmented by a scalar certainty field $c$. That is, $c$ would be identically zero in regions that could not contain the objects, and could range up to one in areas where the objects are likely to exist. $c$ can be constructed from training datasets, from specification by the user, or from an anatomical atlas. The tensor becomes

$$
T_{c}=c T_{\Omega} .
$$

For the case of bone segmentation in a skull CT image, it is difficult for an active contour model to distinguish the bone boundary from the skin boundary which also has high intensity gradients. However, since bone appears bright in $\mathrm{CT}$, it is unlikely to be present in dark areas. Therefore, we construct a binary mask by thresholding the raw dataset with a threshold that is low enough to capture the entire bone structure. This mask restricts the surface evolution to valid locations. Note that direct thresholding of the raw dataset would create artificial boundaries which would severely corrupt the segmentation; however, acting directly on the auxiliary vector field does not introduce new gradients. Figure 4 
shows the ability to segment bone from CT using active contours in conjunction with this spatial prior. The initialization surface is shown first, followed by the unconstrained evolution and the evolution constrained by $c$. The unconstrained evolution is attracted to all high gradients so finds the skin boundary, while the constrained evolution, is able to obtain the bone surface.

\section{Conclusions}

We have introduced adaptive capabilities into the evolution equations for level set based active contour models. These capabilities include modification of the regularization force and stabilization of the image-based auxiliary vector field, both of which are based on the codimension of the signal. That is, they depend on how similar the signal is to a line and to a plane. Additionally, we have introduced the option to spatially constrain the surface evolution by altering this vector field. We predict that these modifications can potentially produce more robust segmentations of medical datasets. In this paper we have demonstrated the feasibility of incorporating local structure information into the evolution equations for level set based active contour models. However much more work is needed in order to test the method on large data sets and to improve the current implementation.

\section{Acknowledgments}

Carl-Fredrik Westin was funded by CIMIT and NIH grants P41-RR13218 and R01-RR11747. Liana Lorigo was funded by NSF Contract IIS-9610249, NSF Contract DMS-9872228, and NSF ERC (Johns Hopkins University agreement) 8810-274. We gratefully acknowledge Renaud Keriven of ENPC, France for efficient level set prototype code. We thank Peter Everett of Brigham and Women's Hospital for making available the CT dataset.

\section{References}

[1] Luigi Ambrosio and Halil M. Soner. Level set approach to mean curvature flow in arbitrary codimension. J. of Diff. Geom., 43:693-737, 1996.

[2] J. Bigün, G. H. Granlund, and J. Wiklund. Multidimensional orientation: texture analysis and optical flow. IEEE Transactions on Pattern Analysis and Machine Intelligence, PAMI-13(8), August 1991.

[3] V. Caselles, F. Catte, T. Coll, and F. Dibos. A geometric model for active contours. Numerische Mathematik, 66:1-31, 1993.

[4] Vicent Caselles, Ron Kimmel, and Guillermo Sapiro. Geodesic active contours. Int'l Journal Comp. Vision, 22(1):61-79, 1997.

[5] Y.G. Chen, Y. Giga, and S. Goto. Uniqueness and existence of viscosity solutions of generalized mean curvature flow equations. J. Differential Geometry, 33:749786, 1991.

[6] L.C. Evans and J. Spruck. Motion of level sets by mean curvature: I. Journal of Differential Geometry, 33:635-681, 1991. 
[7] W. Förstner. A feature based correspondence algorithm for image matching. Int. Arch. Photogrammetry Remote Sensing, 26(3):150-166, 1986.

[8] M. Kass, A. Witkin, and D. Terzopoulos. Snakes: Active contour models. Int J. on Computer Vision, 1(4):321-331, 1988.

[9] A. Kichenassamy, A. Kumar, P. Olver, A. Tannenbaum, and A. Yezzi. Gradient flows and geometric active contour models. In Proc. IEEE Int'l Conf. Comp. Vision, pages 810-815, 1995.

[10] H. Knutsson. Representing local structure using tensors. In The 6th Scandinavian Conference on Image Analysis, pages 244-251, Oulu, Finland, June 1989.

[11] H. Knutsson, H. Bårman, and L. Haglund. Robust orientation estimation in 2D, 3D and 4D using tensors. In Proceedings of Second International Conference on Automation, Robotics and Computer Vision, ICARCV'92, Singapore, September 1992.

[12] T.M. Koller, G. Gerig, G. Szekely, and D. Dettwiler. Multiscale detection of curvilinear structures in $2 \mathrm{D}$ and $3 \mathrm{D}$ image data. In Proc. ICCV'95, pages 864 869, 1995.

[13] L. Lorigo, O. Faugeras, W.E.L. Grimson, R. Keriven, R. Kikinis, and C.-F. Westin. Codimension-Two Geodesic Active Contours. In Proc. IEEE Conf. Comp. Vision and Pattern Recognition, 2000.

[14] R. Deriche O. Monga, R. Lengagne. Extraction of zero crossings of the curvature derivatives in volumetric $3 \mathrm{D}$ medical images: a multi-scale approach. In Proc. IEEE Conf. Comp. Vision and Pattern Recognition, pages 852-855, Seattle, Washington, USA, June 1994.

[15] S. Osher and J. Sethian. Fronts propagating with curvature-dependent speed: Algorithms based on Hamilton-Jacobi formulation. Journal of Computational Physics, 79(1):12-49, 1988.

[16] K. Rohr. Extraction of 3D anatomical point landmarks based on invariance principles. Pattern Recognition, 32:3-15, 1999.

[17] Y. Sato, S. Nakajima, N. Shiraga, H. Atsumi, S. Yoshida, T. Koller, G. Gerig, and R. Kikinis. Three-dimensional multiscale line filter for segmentation and visualization of curvilinear structures in medical images. Medical Image Analysis, 2(2):143-168, 1998.

[18] C.-F. Westin, A. Bhalerao, H. Knutsson, and R. Kikinis. Using Local 3D Structure for Segmentation of Bone from Computer Tomography Images. In Proc. IEEE Conf. Comp. Vision and Pattern Recognition, pages 794-800, Puerto Rico, June 1997.

[19] C.-F. Westin, S.E. Maier, B. Khidhir, P. Everett, F.A. Jolesz, and R. Kikinis. Image Processing for Diffusion Tensor Magnetic Resonance Imaging. In Medical Image Computing and Computer-Assisted Intervention, pages 441-452, September 1999.

[20] C.-F. Westin, J. Richolt, V. Moharir, and R. Kikinis. Affine adaptive filtering of CT data. Medical Image Analysis, 4(2):161-172, 2000.

[21] C.-F. Westin, S. Warfield, A. Bhalerao, L. Mui, J. Richolt, and R. Kikinis. Tensor Controlled Local Structure Enhancement of CT Images for Bone Segmentation. In Medical Image Computing and Computer-Assisted Intervention, pages 1205-1212. Springer Verlag, 1998.

[22] X. Zeng, L. H. Staib, R. T. Schultz, and J. Duncan. Segmentation and measurement of the cortex from 3D mr images. In Medical Image Computing and Computer Assisted Intervention (MICCAI), Boston USA, 1998. 